\title{
Verteilung, Determinanten und Referenzwerte linksventrikulärer Parameter in der Allgemeinbevölkerung - Resultate der MONICA/KORA-Echokardiographiestudien
}
B. Kuch ${ }^{1,5}$
H. Schunkert ${ }^{2}$
M. Muscholl ${ }^{3}$
A. Döring ${ }^{4}$
W. von Scheidt ${ }^{1}$
H.-W. Hense ${ }^{5}$
für die MONICA/KORA-Studien-
gruppe*

\author{
Distribution, Determinants and Reference Values of Left Ventricular Parameters \\ in the General Population - Results of the MONICA/KORA Echocardiography \\ Studies
}

\section{Zusammenfassung}

Voraussetzung für die erfolgreiche Bekämpfung der hohen Mortalität an Herz-Kreislauf-Erkrankungen ist die Erkennung der zugrunde liegenden Risikofaktoren und deren Gewichtung im Hinblick auf Morbidität und Mortalität. In der vorliegenden Arbeit werden deshalb Untersuchungen zusammengefasst, in denen im Rahmen verschiedener MONICA/KORA-Studien (Subpopulationen von S1 bzw. S3) in Augsburg an insgesamt mehr als 2000 Probanden die echokardiographisch ermittelte linksventrikuläre Masse (LVM) und andere linksventrikuläre Parameter und ihre Beziehungen zu Blutdruck und Übergewicht unter besonderer Berücksichtigung von geschlechtsspezifischen Faktoren und Indices der „body size“ untersucht wurden. Hierbei zeigte sich, dass insbesondere Hypertonie und Adipositas für die Entstehung einer linksventrikulären Hypertrophie (LVH) von entscheidender Bedeutung sind. Frauen reagierten - ausgeprägter als Männer insbesondere bei gleichzeitigem Vorliegen von Übergewicht und Hypertonie mit einer konzentrischen Hypertrophie. Bei Anwendung der LV-Hypertrophie(LVH)-Grenzwerte auf eine große Anzahl von Probanden aus der Allgemeinbevölkerung (repräsentative Stichprobe) zeigte sich eine relativ hohe Prävalenz der LVH in der 25- bis 74-jährigen Allgemeinbevölkerung (17,5\% bzw. 18,5\% bei Männern und Frauen). Dies verdeutlicht die hohe prä-

\section{Abstract}

For successful fighting against the burden of cardiovascular disease in the community a comprehensive knowledge about the prevalence and the impact of underlying risk factors is important. The present paper summarises some studies undertaken on more than 2000 persons from the MONICA/KORA-studies (parts of S1 and S3) where left ventricular mass (LVM) and other left ventricular parameters were determined by echocardiography. We especially investigated the associations of LVM with blood pressure and obesity. A special focus was on sex-specific factors in the determination of LVM and the influence of different indices of body size when normalising LVM. It could be shown that hypertension and obesity are major determinants of LV hypertrophy. Especially women with both hypertension and obesity on showed a high prevalence of concentric hypertrophy, significantly more than men. We also showed that the prevalence of LV hypertrophy in a representative sample of the general population ( 25 to 74 years) is $17.5 \%$ for men, and $18.5 \%$ for women. This underscores the need for primary and secondary prevention regarding the development of LV hypertrophy. Finally, our data in a selected group of normal subjects (reference sample) may be used for the development of reference values for left ventricular parameters in the general German population.

Anmerkung

*Die MONICA/KORA-Studiengruppe besteht aus: KORA: H.-E. Wichmann (Sprecher), H. Löwel, C. Meisinger, T. Illig, R. Holle, J. John und deren Mitarbeitern, die verantwortlich für das Design und die Durchführung der KORA-Studien sind; MONICA: U. Keil (Studienleiter), A. Döring, B. Filipiak, H. W. Hense, H. Löwel, J. Stieber und den Mitarbeitern, die verantwortlich für das Design und die Durchführung der MONICA-Studien waren.

Institutsangaben

${ }^{1}$ Klinikum Augsburg, I. Medizinische Klinik, Augsburg

2 Universitätsklinikum Schleswig-Holstein, Campus Lübeck, Medizinische Klinik II, Lübeck

${ }^{3}$ Klinikum der Universität Regensburg, Klinik und Poliklinik für Innere Medizin II, Regensburg

${ }^{4}$ GSF - Forschungszentrum für Umwelt und Gesundheit, Institut für Epidemiologie, Neuherberg

${ }^{5}$ Universität Münster, Institut für Epidemiologie und Sozialmedizin, Münster

Korrespondenzadresse

Priv. Doz. Dr. Bernhard Kuch • I. Medizinische Klinik, Klinikum Augsburg (Kardiologie, Pneumologie, Intensivmedizin, Endokrinologie), Direktor Prof. Dr. W. von Scheidt · Stenglinstraße $2 \cdot 86156$ Augsburg . E-mail: kuchb@aol.com

Bibliografie

Gesundheitswesen 2005; 67 Sonderheft 1: S68 -S73 @ Georg Thieme Verlag KG Stuttgart • New York DOI 10.1055/s-2005-858245

ISSN 0949-7013 
ventivmedizinische und gesundheitsökonomische Relevanz, die der Verhinderung und adäquaten Behandlung einer LVH auf Bevölkerungsebene zukommt. Schließlich bieten unsere Untersuchungen an einer relativ großen Referenzpopulation von herzgesunden Teilnehmern eine Datenbasis, mit der Normalwerte verschiedener linksventrikulärer Parameter für eine deutsche Allgemeinbevölkerung definiert werden können.

\section{Schlïsselwörter}

Echokardiographie · linksventrikuläre Hypertrophie · Geschlechtsunterschiede · Übergewicht · Hypertonie y words

Echocardiography · left ventricular hypertrophy · gender differences $\cdot$ obesity $\cdot$ hypertension

\section{Einleitung}

Zahlreiche epidemiologische Untersuchungen belegen den $\mathrm{Zu}$ sammenhang zwischen erhöhter linksventrikulärer Masse (LVM) und erhöhter kardiovaskulärer Morbidität und Mortalität [1 -3]. Als wesentliche Determinanten der LVM gelten Indices der individuellen Körpermaße (z.B. Körpergröße oder Körperoberfläche $=$ Body Surface Area, BSA), Geschlecht, Hypertonus und Adipositas [3-6]. Bisher unzureichend untersucht waren andere Ursachen einer linksventrikulären Hypertrophie (LVH), wie familiäre Disposition und geschlechtsspezifische Faktoren in der Adaptation des linken Ventrikels auf unphysiologische Belastungen wie zu hoher Blutdruck und/oder Übergewicht [7, 8]. Die Abhängigkeit der LVM von unterschiedlichen Körpermaßen (im Englischen subsumiert unter dem Begriff „body size“) macht es notwendig, die LVM zu „normalisieren“ und auf ein Maß der „body size“ zu „indexieren“ $[9,10]$. Als optimale Indexierungsmethode wurde hierbei die so genannte "lean body mass“ oder fettfreie Körpermasse (FFM) angesehen, da diese die metabolischen und hämodynamischen Erfordernisse der LVM am besten ausdrückt [11 - 13]. Weiterhin ist es schwierig, bei einer kontinuierlichen Variable wie der LVM oder der Wanddicken bzw. des enddiastolischen Durchmessers festzulegen, wo deren kritische Grenze liegt, ab der man von einer abnormalen Anpassung sprechen kann. Die Festlegung einer Grenze für die Definition einer LVH ist notwendig, da sie auch die jeweilige Prävalenz an LVH in definierten Patienten- oder Allgemeinbevölkerungsgruppen beeinflusst [14]. In der vorliegenden Arbeit werden deshalb einige Untersuchungen zusammengefasst, die im Rahmen verschiedener MONICA/KORA-Studien in Augsburg an insgesamt mehr als 2000 Probanden durchgeführt wurden. Insbesondere wurden Zusammenhänge zwischen der echokardiographisch ermittelten LVM und ihren Beziehungen zu Blutdruck und Übergewicht unter besonderer Berücksichtigung von geschlechtsspezifischen Faktoren und Indices der „body size“ untersucht. Einen Schwerpunkt dieser Arbeit bilden hier insbesondere die Generierung von Grenzwerten für verschiedene Parameter der linksventrikulären Geometrie und die Darstellung, wie sich dies auf die Prävalenz in der repräsentativen Allgemeinbevölkerung auswirkt.

\section{Methodik}

\section{Untersuchte Populationen}

Im Rahmen der vorliegenden Arbeit wurden folgende Populationen untersucht:
- Teilnehmer des ersten MONICA-Augsburg-Surveys S1 (1984/85), die 1987-1988 (K1) nachuntersucht wurden ( $\mathrm{n}=227$ Männer und 293 Frauen, Altersbereich von 52 bis 67 Jahre) $[15,16]$.

- Im Rahmen des dritten MONICA-Augsburg-Surveys S3 (1994/95) wurden 4856 Männer und Frauen (Teilnehmerrate $75 \%$ ) untersucht. Für diejenigen Personen, die in der Stadt Augsburg lebten (Anzahl 2376), wurde eine zusätzliche echokardiographische Untersuchung angeboten. Hiervon nahmen 826 Männer und 852 Frauen teil (repräsentativer Bevölkerungsquerschnitt [17]).

- Gesunde Referenzpopulation aus S3.

Für die Generierung von Referenzwerten der LVM wurde aus dem gesamten Bevölkerungsquerschnitt (S3) eine gesunde Referenzpopulation folgendermaßen generiert: Ausschluss aller Probanden mit unzureichender Qualität der echokardiographischen Untersuchung $(\mathrm{n}=271)$, Ausschluss aller Probanden mit kardiopulmonaler Erkrankung $(\mathrm{n}=776)$, Ausschluss aller Probanden mit arteriellem Hypertonus ( $n=657$ ), Ausschluss aller Probanden mit Medikamenteneinnahme für eine kardiopulmonale Erkrankung $(\mathrm{n}=327)$ und Ausschluss aller Probanden mit Übergewicht (BMI größer als $27,3 \mathrm{~kg} / \mathrm{m}^{2}$ bei Männern und $27,8 \mathrm{~kg} / \mathrm{m}^{2}$ bei Frauen; $\mathrm{n}=660$ ) (Zugehörigkeit zu mehreren Gruppen möglich).

Daraus resultierte schließlich eine Referenzgruppe von 213 Männern und 291 Frauen [17].

\section{Untersuchungen}

Die echokardiographische Aufzeichnung erfolgte unter standardisierten Bedingungen [16, 17]. Alle M-Mode-Aufzeichnungen wurden von einem einzigen versierten Untersucher, dem die klinischen Daten der Probanden nicht bekannt waren, analysiert. Die LVM wurde einerseits gemäß der Penn-Konvention bestimmt und nach der Formel von Devereux und Reichek [18] berechnet und andererseits nach ASE-Konvention bestimmt [19]. Die Berechnungen basieren auf Messungen des linksventrikulären enddiastolischen Durchmessers (LVEDD), der Septumwanddicke (SWD) und der Hinterwanddicke (HWD). Bei der ASE-Konvention werden die Endokardkonturen in die Wanddickenvermessung mit aufgenommen, wohingegen diese bei der Penn-Konvention in die Vermessung des LVEDD einfließen.

Die Bioimpedanzanalyse (BIA) war Bestandteil des Untersuchungsprotokolls der dritten MONICA-Augsburg-Bevölke- 
Hetails zur Bestimmung und der verwendeten Formel wurden berichtet $[17,20]$.

Als Maß für das relative Körpergewicht wurde der Body Mass Index (BMI) verwendet mit BMI = Körpergewicht/Körpergröße ${ }^{2}\left(\mathrm{~kg} / \mathrm{m}^{2}\right)$. Es wurden folgende Gruppierungen vorgenommen: Schlank bei $\mathrm{BMI}<23 \mathrm{~kg} / \mathrm{m}^{2}$, leicht übergewichtig bei BMI von $23-25,99 \mathrm{~kg} / \mathrm{m}^{2}$, mäßig übergewichtig bei BMI von $26-29,99 \mathrm{~kg} / \mathrm{m}^{2}$ und sehr übergewichtig bei BMI von $\geq 30 \mathrm{~kg} / \mathrm{m}^{2}$ bzw. (Abb.1) normalgewichtig (N) bei $B M I<25 \mathrm{~kg} / \mathrm{m}^{2}$, mild übergewichtig (OB) bei BMI von $25-29,99 \mathrm{~kg} / \mathrm{m}^{2}$ und deutlich übergewichtig (OBS) bei BMI von $\geq 30 \mathrm{~kg} / \mathrm{m}^{2}$. Die Unterteilung in verschiedene Blutdruckklassen erfolgte in: unbehandelt hyperton $(U)$ bei systolischen Werten $\geq 160 \mathrm{mmHg}$ oder diastolisch $\geq 95 \mathrm{mmHg}$ ohne antihypertensive Medikation, behandelt hyperton $(\mathrm{TH})$, wenn antihypertensive Medikation bei bekannter Hypertonie eingenommen wurde, grenzwertig (Borderline) hyperton (B) bei systolischen Werten zwischen 140 und $159 \mathrm{mmHg}$ und diastolischen Werten zwischen 90 und $94 \mathrm{mmHg}$ und normoton $(\mathrm{N})$ bei Werten unter 140 resp. $90 \mathrm{mmHg}$.

\section{Statistik}

Der $\chi^{2}$-Test und der Students-t-Test wurden verwendet, um kategorische Variablen bzw. Mittelwerte von kontinuierlichen Variablen zu testen. Univariate lineare Assoziationen zwischen linksventrikulären Parametern, Blutdruck und anthropometrischen Parametern wurden mittels Pearson-Korrelationskoeffizienten berechnet. Um die statistische Signifikanz der Mittelwerte der LV-Parameter in Abhängigkeit der verschiedenen Gewichts- und Blutdruckklassen auf Alter und jeweils BMI bzw. systolischen und diastolischen Blutdruck zu adjustieren, wurden multivariate lineare Regressionsmodelle verwendet.

Alle statistischen Analysen wurden mit dem SAS ${ }^{\circledR}$-System für Windows 6.10 durchgeführt.

\section{Ergebnisse}

Es zeigten sich bei den 52- bis 67-jährigen Männern und Frauen $(\mathrm{n}=520)$ jeweils unabhängige und bei beiden Geschlechtern aus-
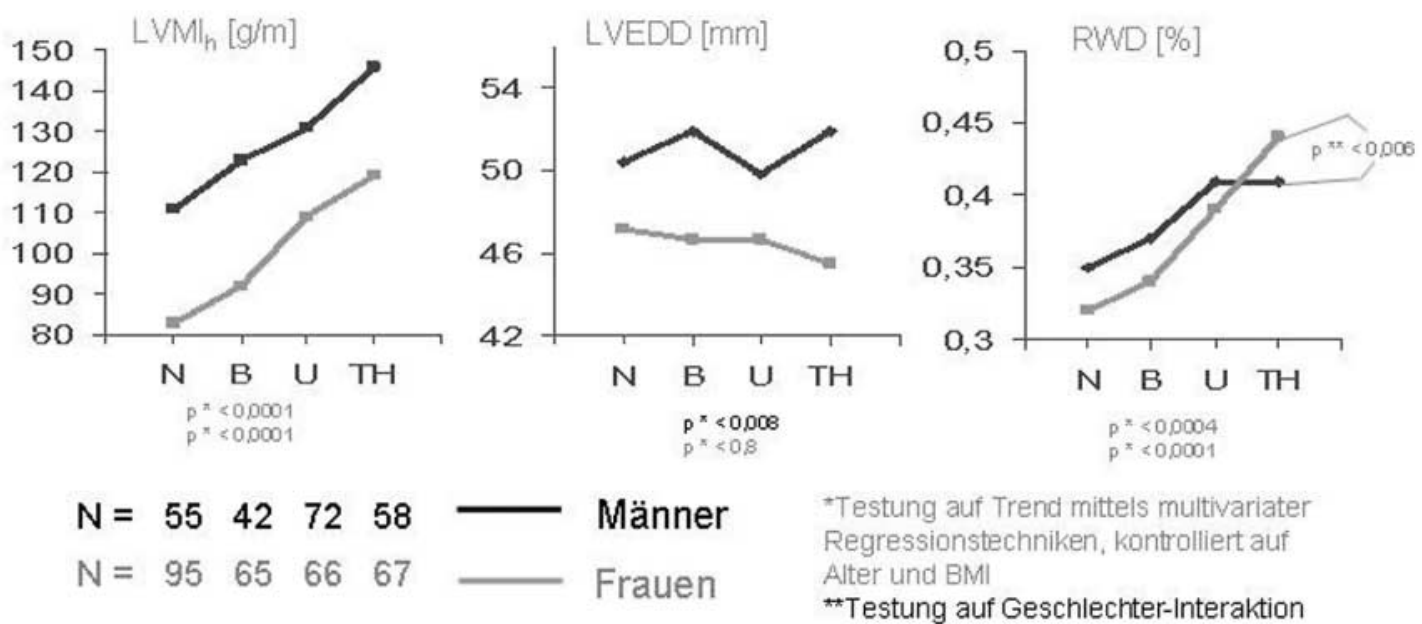

Abb. 1 Einflüsse steigender Blutdruckgrade auf die auf Körpergröße indexierte linksventrikuläre Masse (LVMIh), den linksventrikulären enddiastolischen Durchmesser (LVEDD) und die relative Wanddicke
(RWD); $\mathrm{N}$ = normotensiv, $\mathrm{B}=$ Borderline-hypertensiv, $\mathrm{U}=$ unbehandelt hypertensiv, $\mathrm{TH}$ = behandelt hypertensiv; MONICA-Augsburg-Echokardiographiestudien. 

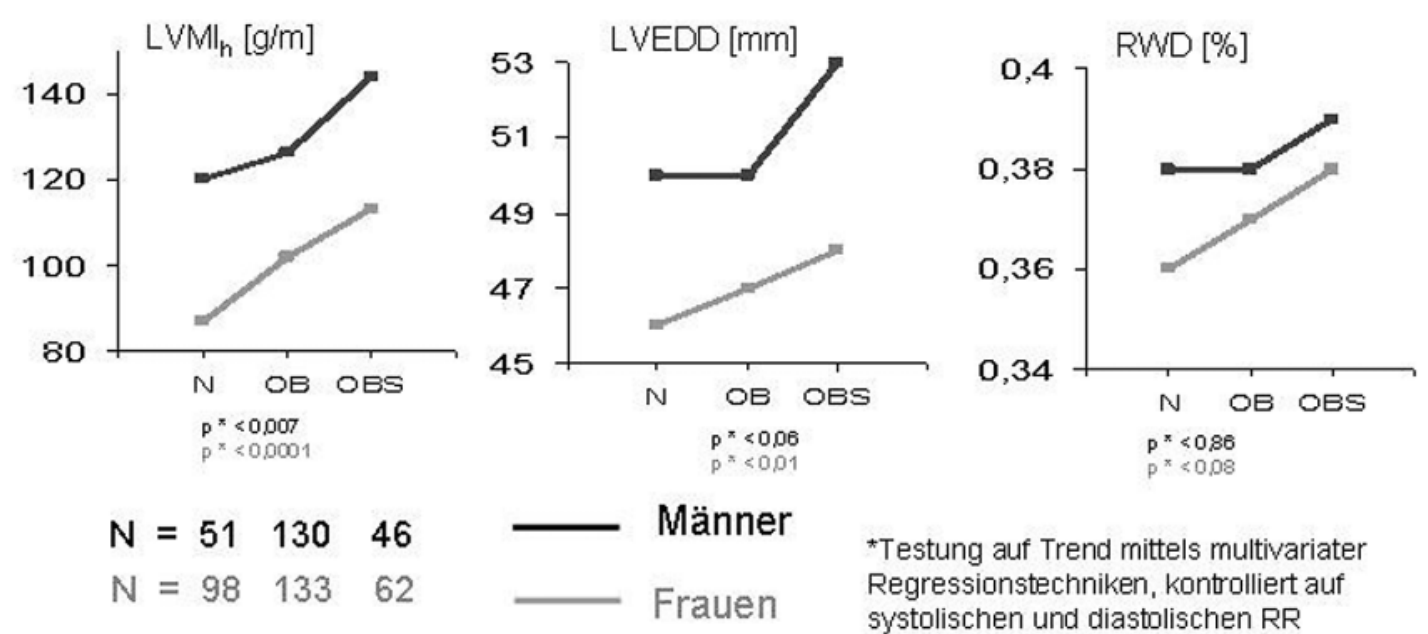

*Testung auf Trend mittels multivariater
Regressionstechniken, kontrolliert auf

systolischen und diastolischen RR

Abb. 2 Einflüsse steigender Übergewichtsgrade auf die auf Körpergröße indexierte linksventrikuläre Masse (LVMIh), den linksventrikulären enddiastolischen Durchmesser (LVEDD) und die relative Wand- dicke (RWD); $\mathrm{N}=$ normalgewichtig, $\mathrm{OB}=$ mild übergewichtig, OBS = deutlich übergewichtig; MONICA-Augsburg-Echokardiographiestudien.
Ausnahme der externen FFM-basierten LVH-Kriterien waren mit allen Indexierungen höhere LVH-Prävalenzen bei Frauen im Vergleich zu Männern zu verzeichnen. Allerdings waren diese Geschlechtsunterschiede für keine der aus internen Kriterien ermittelten LVH-Prävalenzen signifikant [17].

Durch Generierung einer gesunden Referenzpopulation konnten erstmalig für die deutsche Allgemeinbevölkerung im Alter von 45 bis 74 Jahren Grenzwerte für erhöhte echokardiographische Parameter abgeleitet werden. In Tab. 1 sind alle Grenzwerte, ermittelt als Mittelwert + 2 Standardabweichungen, für die Dicken von Hinterwand und Septum, den LVEDD, die unindexierte LVM und für die jeweiligen indexierten LVM für Messungen nach Penn sowie der ASE-Konvention dargestellt. Die Grenzwerte stellen sich dabei als sehr stark abhängig vom Geschlecht und der Indexierungsmethode der LVM heraus. Generell waren die LVMGrenzwerte basierend auf Indexierung mit Körpergröße, Grö$ß^{2,7}$ und BSA für Männer höher als für Frauen. Hingegen ergaben sich identische Grenzwerte für Männer und Frauen bei Indexierung der LVM auf die FFM [20, 25].

\section{Diskussion}

Die generell sowohl bei Frauen wie bei Männern beobachtete Dominanz an exzentrischer Hypertrophie bei Übergewicht und konzentrischer Hypertrophie bei Vorliegen von Hypertonie wurde aufgrund pathophysiologischer Überlegungen schon länger postuliert und auch schon in früheren Studien beobachtet [5, 22, 23]. Ein neuer Aspekt unserer Untersuchungen ist hierbei, dass es in Zusammenhang mit erhöhtem Blutdruck geschlechtsspezifische Reaktionsweisen mit verstärktem Anstieg an konzentrischer Hypertrophie bei Frauen gibt. Diese geschlechtsspezifischen Zusammenhänge wurden bisher erst wenig untersucht [7, 8]. Es müssen also bei gleichen Bedingungen lastabhängiger Faktoren (unterschiedliche Hypertonie- und Übergewichtsgrade) Einflüsse lastunabhängiger Faktoren vorliegen, die für diese Geschlechtsunterschiede verantwortlich sind. Mögliche hierfür zugrunde liegende Mechanismen sind Geschlechtsunterschiede in der kardialen

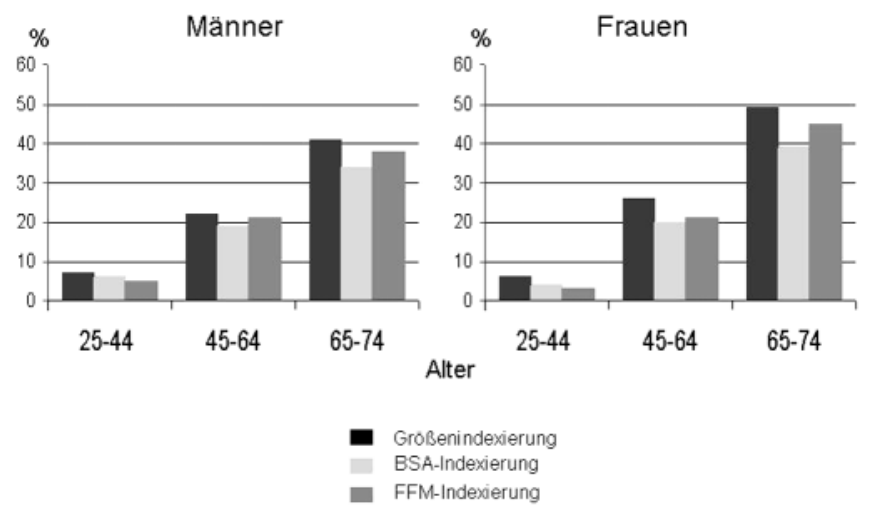

Abb. 3 Prävalenz der linksventrikulären Hypertrophie (LVH) in der Allgemeinbevölkerung (repräsentativer Bevölkerungsquerschnitt $n=1,371$ ) in Abhängigkeit vom Alter und der Indexierungsmethode der LVM; BSA = Body Surface Area, FFM = fettfreie Masse; MONICAAugsburg-Echokardiographiestudien.

Genexpression als Reaktion z. B. auf Druckbelastung [23]. Für hormonelle Einflüsse sprechen auch Zusammenhänge mit der Menopause (mehr als 90\% der Frauen der ersten untersuchten Studienpopulation befanden sich in der Postmenopause). Tierversuche bieten Hinweise für einen „Schutzeffekt“ der Östrogene, der postmenopausal durch ein relatives Überwiegen der Androgene ersetzt wird [26, 27]. Auch das Renin-Angiotensin-Aldosteron-System scheint geschlechtsspezifisch auf die Entwicklung einer LVH Einfluss zu nehmen, wie unterschiedlich starke LVH-Assoziationen von Serum-ACE-Aktivität und Aldosteron bei Männern und Frauen belegen [28].

Die in der Augsburger Bevölkerung beobachtete verstärkte Ausbildung einer konzentrischen LVH bei Frauen mit Hypertonie, und verstärkt bei gleichzeitigem Vorliegen von Hypertonie und Übergewicht, ist von besonderer Bedeutung, da gezeigt werden konnte, dass die konzentrische Hypertrophie von allen Hypertrophieformen mit der schlechtesten Prognose verbunden ist [29-32]. In verschiedenen Studien wurde beschrieben, dass Frauen mit einer LVH eine schlechtere Prognose aufweisen als 
Tab. 1 Grenzwerte linksventrikulärer Parameter ermittelt als Mittelwert (MW) + 2 Standardabweichungen (SD) nach Penn sowie der ASEKonvention; gesunde Referenzpopulation aus MONICA Augsburg (Subpopulation von S3), Alter 25 bis 74 Jahre

\begin{tabular}{|c|c|c|c|c|}
\hline & \multicolumn{2}{|c|}{ Männer (n = 213) } & \multicolumn{2}{|c|}{ Frauen $(n=291)$} \\
\hline & MW & $\begin{array}{l}M W \\
+2 S D\end{array}$ & $M W$ & $\begin{array}{l}M W \\
+2 S D\end{array}$ \\
\hline $\begin{array}{l}\text { SWD (mm) } \\
\text { (ASE) }\end{array}$ & 10 & 12 & 9 & 11 \\
\hline $\begin{array}{l}\text { SWD (mm) } \\
\text { (Penn) }\end{array}$ & 9 & 11 & 8 & 11 \\
\hline $\begin{array}{l}\mathrm{HWD}(\mathrm{mm}) \\
\text { (ASE) }\end{array}$ & 8 & 11 & 7 & 9 \\
\hline $\begin{array}{l}\text { HWD (mm) } \\
\text { (Penn) }\end{array}$ & 7 & 10 & 7 & 9 \\
\hline $\begin{array}{l}\operatorname{LVEDD}(\mathrm{mm}) \\
\text { (ASE) }\end{array}$ & 50 & 58 & 45 & 52 \\
\hline $\begin{array}{l}\text { LVEDD (mm) } \\
\text { (Penn) }\end{array}$ & 52 & 60 & 48 & 54 \\
\hline $\begin{array}{l}\text { RWD } \\
\text { (ASE) }\end{array}$ & 0,37 & 0,48 & 0,36 & 0,48 \\
\hline $\begin{array}{l}\text { RWD } \\
\text { (Penn) }\end{array}$ & 0,29 & 0,41 & 0,29 & 0,40 \\
\hline $\begin{array}{l}\operatorname{LVM}(\mathrm{g}) \\
\text { (ASE) }\end{array}$ & 159 & 215 & 114 & 156 \\
\hline $\begin{array}{l}\operatorname{LVM}(\mathrm{g}) \\
\text { (Penn) }\end{array}$ & 171 & 247 & 117 & 146 \\
\hline $\begin{array}{l}\text { LVM/Größe }(\mathrm{g} / \mathrm{m}) \\
\text { (ASE) }\end{array}$ & 90 & 120 & 70 & 95 \\
\hline $\begin{array}{l}\text { LVM/Größe (g/m) } \\
\text { (Penn) }\end{array}$ & 97 & 139 & 71 & 108 \\
\hline $\begin{array}{l}\text { LVM/Größe }\left(\mathrm{g} / \mathrm{m}^{2}\right) \\
\text { (ASE) }\end{array}$ & 51 & 68 & 43 & 59 \\
\hline $\begin{array}{l}\operatorname{LVM}_{\text {(Vröße }} \text { (g/m²) } \\
\text { (Penn) }\end{array}$ & 55 & 78 & 44 & 67 \\
\hline $\begin{array}{l}\mathrm{LVM} / G r o ̈ ß e^{2,7}\left(\mathrm{~g} / \mathrm{m}^{2,7}\right) \\
\text { (ASE) }\end{array}$ & 34 & 46 & 31 & 42 \\
\hline $\begin{array}{l}\text { LVM/Größe }{ }^{2,7}\left(\mathrm{~g} / \mathrm{m}^{2,7}\right) \\
\text { (Penn) }\end{array}$ & 37 & 53 & 31 & 48 \\
\hline $\begin{array}{l}\operatorname{LVM} / B S A\left(\mathrm{~g} / \mathrm{m}^{2}\right) \\
\text { (ASE) }\end{array}$ & 82 & 108 & 69 & 92 \\
\hline $\begin{array}{l}\text { LVM/BSA (g/m²) } \\
\text { (Penn) }\end{array}$ & 89 & 125 & 70 & 104 \\
\hline $\begin{array}{l}\text { LVM/FFM (g/kg) } \\
\text { (ASE) }\end{array}$ & 2,7 & 3,6 & 2,7 & 3,6 \\
\hline $\begin{array}{l}\text { LVM/FFM (g/kg) } \\
\text { (Penn) }\end{array}$ & 2,9 & 4,1 & 2,7 & 4,1 \\
\hline
\end{tabular}

Männer [2, 33]. Dies könnte damit verbunden sein, dass Frauen eher dazu neigen, auf entsprechende Belastungen mit konzentrischer Ventrikelanpassung zu reagieren.

Erstmalig wurde auch an einer großen deutschen Bevölkerungsstichprobe die LVH-Prävalenz aufgezeigt. Unsere Analysen zeigten, dass die ungeprüfte Anwendung externer, d.h. aus anderen Populationen generierter Kriterien zu ausgeprägt fehlerhaften Prävalenzberechnungen führen kann und dass, wenn immer möglich intern, d.h. in den jeweiligen Bevölkerungen entwickelte Kriterien zur Anwendung kommen sollten.

Wir konnten erstmalig für eine deutsche Allgemeinbevölkerung Grenzwerte für linksventrikuläre Parameter auch unter Berücksichtigung unterschiedlicher Indexierungsmethoden und unter- schiedlicher Methodik in der Bestimmung (ASE- oder Penn-Konvention) der LVM für die Definition einer LVH entwickeln. Diese könnten als Basis für die Anwendung von Grenzwerten linksventrikulärer Parameter in Studien und im klinischen Alltag herangezogen werden. Sie differieren zum Teil deutlich von Referenzwerten aus der Framingham-Studie.

\section{Bedeutung/Ausblick}

Voraussetzung für die erfolgreiche Bekämpfung der hohen Mortalität an Herz-Kreislauf-Erkrankungen ist die Erkennung der zugrunde liegenden Risikofaktoren und deren Gewichtung im Hinblick auf Morbidität und Mortalität. Anhand der vorliegenden Daten ist zur Verhinderung einer LVH eine intensive Prophylaxe und/oder Behandlung von Übergewicht und Hypertonus zu fordern. Die relativ hohe Prävalenz der LVH in der 25- bis 74-jährigen Allgemeinbevölkerung (17,5\% bzw. 18,5\% bei Männern und Frauen, FFM) verdeutlicht die hohe präventivmedizinische und gesundheitsökonomische Relevanz, die der Verhinderung und adäquaten Behandlung einer LVH auf Bevölkerungsebene zukommt.

Im Rahmen der Augsburger Diabetes-Familienstudie, deren Ziel die Identifikation und Charakterisierung von Genen ist, die zu der Entwicklung des Typ-2-Diabetes verschiedener phänotypischer Ausprägungen beitragen, wurden an 787 Probanden echokardiographische Untersuchungen durchgeführt. Erste Analysen bei Probanden mit manifestem Diabetes zeigen eine hohe Prävalenz an systolischen Funktionsstörungen bei bisher hinsichtlich einer kardiovaskulären Erkrankung asymptomatischen Patienten sowie eine auffällig hohe Prävalenz an diastolischer Funktionsstörung bei allen Teilnehmern (Publikation in Vorbereitung).

Im Rahmen einer groß angelegten Follow-up-Untersuchung F3 aller S3-Teilnehmer werden seit Februar 2004 (geplantes Studienende April 2005) unter anderem echokardiographische Nachuntersuchungen aller echokardiographisch untersuchten Probanden durchgeführt. Hierbei werden verschiedenste Fragestellungen bezüglich der Inzidenz und Determinanten einer LVH bei vorher Gesunden untersucht. Im Rahmen dessen findet auch ein Morbiditäts- und Mortalitäts-Follow-up aller S3-Teilnehmer statt, das prognostische Aussagen bezüglich verschiedener Gruppen von LVH-Patienten erlauben wird. Darüber hinaus soll untersucht werden, wie sich die Anwendung verschiedener Indexierungsmethoden auf das Outcome auswirkt.

\section{Danksagung}

Die MONICA/KORA-Studien zur LVH wurden unterstützt von der GSF und vom BMBF - Bundesministerium für Forschung und Technologie (FKZ KBF 01 GB 9403 und Nationales Genom Forschungsnetz NGFN) und der DFG - Deutschen Forschungsgemeinschaft (Schu 672/9-1, Schu 672/10-1).

Der Artikel nimmt besonders Bezug auf folgende Beiträge dieses Sonderheftes von „Das Gesundheitswesen“ [34-36]. 
${ }^{19}$ Devereux RB, Koren MJ, deSimone P et al. Methods for detection of left ventricular hypertrophy: application to hypertensive heart disease. Eur Heart J 1993; 14 (Suppl D): 8-15

${ }^{1}$ Casale PN, Devereux RB, Milner M et al. Value of echocardiographic left ventricular mass in predicting cardiovascular morbid events in hypertensive men. Ann Intern Med 1986; 105: 173 - 178

2 Levy D, Garrison RJ, Savage DD et al. Prognostic implications of echocardiographically determined left ventricular mass in the Framingham Heart Study. N Engl J Med 1990; 322: 1561 - 1566

${ }^{3}$ Cooper RS, Simmons BE, Castaner A et al. Left ventricular hypertrophy is associated with worse survival independent of ventricular function and number of coronary arteries severely narrowed. Am J Cardiol 1990; 65: $441-445$

${ }^{4}$ Devereux RB, Roman MJ, de Simone G et al. Relations of left ventricular mass to demographic and hemodynamic variables in American Indians. The Strong Heart Study. Circulation 1997; 96: 1416-1423

${ }^{5}$ Hammond IW, Devereux RB, Aldermann MH et al. Relation of Blood Pressure and Body Build to Left Ventricular Mass in Normotensive and Hypertensive Employed Adults. J Am Coll Cardiol 1988; 12: 996- 1004

${ }^{6}$ Liebson RP, Grandits G, Prineas R et al. Echocardiographic correlates of left ventricular structure among 844 mildly hypertensive men and women in the treatment of mild hypertension study (TOMHS). Circulation 1993; 87: $476-486$

${ }^{7}$ Aurigemma GP, Gaasch WH. Gender differences in older patients with pressure-overload hypertrophy of the left ventricle. Cardiology 1995; 86: $310-317$

8 Krumholz HM, Larson M, Levy D. Sex differences in cardiac adaptation to isolated systolic hypertension. Am J Cardiol 1993; 72: 310 - 313

${ }^{9}$ Levy D, Anderson KM, Savage DD et al. Echocardiographically Detected Left Ventricular Hypertrophy: Prevalence and Risk Factors. Ann Intern Med 1988; 108: 7-13

${ }^{10}$ Levy D, Savage DD, Garrison RJ et al. Echocardiographic Criteria for Left Ventricular Hypertrophy: The Framingham Heart Study. Am J Cardiol 1987; 59: $956-960$

${ }^{11}$ Bella JN, Devereux RB, Roman MJ et al. Relations of left ventricular mass to fat-free and adipose mass. The Strong Heart Study. Circulation 1998; 98: $2538-2544$

${ }^{12}$ Daniels SR, Kimball TR, Morrison JA et al. Indexing left ventricular mass to account for differences in body size in children and adolescents without cardiovascular disease. J Am Coll Cardiol 1995; 76: 699-701

${ }^{13}$ Whalley GA, Gamble GD, Doughty RN et al. Left ventricular mass correlates with fat-free mass but not fat mass in adults. J Hypertens 1999; 17: $569-574$

${ }^{14}$ Abergel E, Tase M, Bohlender J et al. Which Definition for Echocardiographic Left Ventricular Hypertrophy? Am J Cardiol 1995; 75: $498-502$

${ }^{15}$ Keil U, Filipiak B, Doring A et al. Monitoring of trends and determinants in cardiovascular disease in Germany: Results of the MONICA Project Augsburg, 1985-1990. MMWR Morb Mortal Wkly Rep 1992; 41 (Suppl): $171-179$

${ }^{16}$ Kuch B, Muscholl M, Luchner A et al. Geschlechtsunterschiede in der Beziehung zwischen Übergewicht und Hypertonie mit linksventrikulärer Masse und Hypertrophie. Zeitschrift für Kardiologie 1996; 85: $334-342$

${ }^{17}$ Kuch B, Hense HW, Gneiting B et al. Body Composition and Prevalence of Left Ventricular Hypertrophy. Circulation 2000; 102: 405-410

${ }^{18}$ Devereux RB, Reichek N. Echocardiographic determination of left ventricular mass in man: anatomic validation of the method. Circulation 1977; 55: 613-618

${ }^{20}$ Hense HW, Gneiting B, Muscholl M et al. The associations of body size and body composition with left ventricular mass: impact for indexation in adults. J Am Coll Cardiol 1998; 32: $451-457$

${ }^{21}$ Kuch B, Muscholl M, Luchner A et al. Gender specific differences in left ventricular adaptation to obesity and hypertension. Journal of Human Hypertension 1998; 12: 685-691

22 Garavaglia GE, Messerli FH, Schmieder RE et al. Myocardial contractility and left ventricular function in obese patients with essential hypertension. Am J Cardiol 1988; 62: 594-597

${ }^{23}$ Gottdiener JS, Reda DJ, Materson BJ et al. Importance of obesity, race and age to the cardiac structural and functional effects of hypertension. J Am Coll Cardiol 1994; 24: 1492 - 1498

24 de Simone G, Daniels SR, Devereux RB et al. Left Ventricular Mass and Body Size in Normotensive Children and Adults: Assessment of Allometric Relations and Impact of Overweight. J Am Coll Cardiol 1992; 20: $1251-1260$

25 Kuch B, Gneiting B, Döring A et al. Indexation of Left Ventricular Mass in Adults with a Novel Approximation for Fat-free Mass. J Hypertens 2001; 19: $135-142$

${ }^{26}$ Cabral AM, Vasquez EC, Moyses MR et al. Sex hormone modulation of ventricular hypertrophy in sinoaortic denervated rats. J Hypertens 1984; $2: 85-91$

${ }^{27}$ König H, Goldstone A, Lu CY. Testosteron mediated sexual dimorphism of the rodent heart, ventricular lysosomes, mitochondria and cell growth as modulated by androgens. Cir Res 1982; 50: 782 - 787

28 Schunkert H, Hense HW, Muscholl M et al. Associations between circulating components of the renin-angiotensin-aldosterone system and left ventricular mass. Heart 1997; 77: 24-31

${ }^{29}$ Aranow W, Ahn C, Kronzon I et al. Congestive heart failure, coronary events and atherothrombotic brain infarction in elderly blacks and whites with systemic hypertension and with and without echocardiographic and electrocardiographic evidence of left ventricular hypertrophy. Am J Cardiol 1991; 67: 295 - 299

${ }^{30}$ Ghali JK, Liao Y, Cooper RS. Influence of left ventricular geometric patterns on prognosis in patients with or without coronary artery disease. J Am Coll Cardiol 1998; 31: 1635-1640

31 Koren MJ, Devereux RB, Casale PN et al. Relation of left ventricular mass and geometry to morbidity and mortality in uncomplicated essential hypertension. Ann Intern Med 1991; 114: 345-352

32 Krumholz HM, Larson M, Levy D. Prognosis of left ventricular geometric patterns in the Framingham Heart Study. J Am Coll Cardiol 1995; $25: 879-884$

${ }^{33}$ Liao Y, Cooper RS, Mensah GA et al. Left ventricular hypertrophy has a greater impact on survival in women than in men. Circulation 1995; 92: $805-810$

${ }^{34}$ Löwel H, Döring A, Schneider A et al. The MONICA Augsburg surveys basis for prospective cohort studies. Gesundheitswesen 2005; 67 S1: S13-S18

${ }^{35}$ Holle R, Happich M, Löwel H et al. KORA - A research platform for population based health research. KORA - Eine Forschungsplattform für bevölkerungsbezogene Gesundheitsforschung. Gesundheitswesen 2005; 67 S1: S19-S25

${ }^{36}$ Kuch B, Schunkert H, Muscholl M et al. Verteilung, Determinanten und Referenzwerte linksventrikulärer Parameter in der Allgemeinbevölkerung - Resultate der MONICA/KORA Echokardiographiestudien. Gesundheitswesen 2005; 67 S1: S68 -S73 